\title{
Magnetic Resonance Imaging of Young and Aged Rat Brains under a Magnetic Field of $7.05 \mathrm{~T}$
}

\author{
Mikinori KUWABARA*, Taketoshi ASANUMA, Osamu INANAMI ${ }^{1)}$, Takashi JIN $^{2)}$, Shigezo SHIMOKAWA ${ }^{3)}$, \\ Noriyuki $\mathrm{KASAI}^{4)}$, Kunihiko $\mathrm{KATOR}^{5)}$, and Fumiaki SATO \\ Faculty of Veterinary Medicine, ${ }^{2}$ Institute for Electronic Science, ${ }^{3)}$ Faculty of Engineering, ${ }^{4)}$ School of Medicine, Hokkaido University, \\ Sapporo 060, ${ }^{1)}$ Faculty of Agriculture, Iwate University, Morioka 020, and ${ }^{5)}$ Faculty of Science, The University of Tokyo, Tokyo 113, \\ Japan
}

(Received 14 April 1994/Accepted 1 July 1994)

\begin{abstract}
Using a homemade MR imaging probe (Helmholtz coil), MR images of brains of 5-week-old and 23- or 24-month-old Wistar rats were taken under a magnetic field of $7.05 \mathrm{~T}$ (Tesla). The probe was designed to fit the rat head and made by winding thin copper film round an acrylic tube with a 5-cm i.d., 10-cm length and 2-mm thickness. This was adjusted to resonate with the $300 \mathrm{MHz}$ radiofrequency corresponding to the resonance frequency of ${ }^{1} \mathrm{H}$ under a magnetic field of $7.05 \mathrm{~T}$. MR images were obtained by $\mathrm{T}_{1}$-weighted and two-dimensional Fourier transformation techniques. The sagittal and coronal sections were imaged in 1-mm-thick slices. The size of the data matrix was 128 phase-encoded steps. Each image was obtained through eight acquisitions. A comparison of the MR images with those semi-microscopically taken at the same position of the coronal section revealed that the cerebral cortex, hippocampus, hypothalamus and thalamus were clearly imaged by this probe. With aging, MR images of cerebral cortices were observed with decreased signal intensities. Enlargement of the third ventricles and hypertrophy of cranical parietal bones were also recognized in sagittal MR images of aged rats. These observations were more marked in males than in females. From these observations it was concluded that this probe was applicable for MR imaging of rat brains under a magnetic field of 7.05 T. - KEY woRDS: aging, brain, MRI, MRI probe, rat.
\end{abstract}

J. Vet. Med. Sci. 55(6): 933-938, 1994

Various disease models induced artificially in small laboratory animals such as mice and rats are utilized as models of human diseases. MR imaging method is successfully employed to judge the stages of the diseases in man, however, when the laboratory animals are very small, it is hard to obtain strong NMR signals with a high $\mathrm{S} / \mathrm{N}$ ratio. The NMR theory teaches us that the higher the magnetic field the stronger are the NMR signals obtained [7]. Therefore, experiments concerning MR images of rat brains have been carried out under magnetic fields of $2.0 \mathrm{~T}$ [8] and $4.7 \mathrm{~T}[4,9,14]$, higher than those for humans which are about $0.5-1.5 \mathrm{~T}$. In the present study we tried to obtain MR images of rat brains under a magnetic field of 7.05 T. For this purpose we made a Helmholtz-type MR probe that resonated at $300 \mathrm{MHz}$, corresponding to the resonance frequency of ${ }^{1} \mathrm{H}$ at the magnetic field of $7.05 \mathrm{~T}$, and then this probe was used to examine whether the MR images of brains of aged rats were distinguishable from those of young rats.

MATERIALS AND METHODS

MRI: MR imaging probe (Helmholtz coil design, Dadok type) that resonated at a radiofrequency of 300 $\mathrm{MHz}$ was made of thin copper film (Fig. 1) [7]. An acrylic tube with an i.d. of $5 \mathrm{~cm}$, that was $10 \mathrm{~cm}$ long and $2 \mathrm{~mm}$ thick, was employed as a support of the probe. A teflon

\footnotetext{
* Correspondence to: Kuwabara, M., Department of Radiation Biology, Faculty of Veterinary Medicine, Hokkaido University, Sapporo 060, Japan.
}

sheet with an adequate thickness was inserted between two layers of copper film as an insulator to make the probe resonate at $300 \mathrm{MHz}$ (Figs. 1a and 1b). Proton images were obtained on an Oxford 7.05 T magnet equipped with a Toshiba workstation with an SIS 300/183 imaging system using a two-dimensional Fourier transformation technique. The signal intensity in an imaging study can be expressed as:

$$
\mathrm{I}=\mathrm{KN}\left[1-\exp \left(\mathrm{T}_{\mathrm{R}} / \mathrm{T}_{1}\right)\right] \exp \left(-\mathrm{T}_{\mathrm{E}} / \mathrm{T}_{2}\right)
$$

where $\mathrm{K}$ is a scaling term, $\mathrm{N}$ is the density of hydrogen nuclei, $T_{R}$ is the repetition period for the entire sequence, $T_{E}$ is the echo time, $T_{1}$ is the spin-lattice relaxation time, and $T_{2}$ is the spin-spin relaxation time $[1,3,5,6]$. To obtain the optimum conditions for $\mathrm{T}_{1}$-weighted images, MR images of a phantom consisting of water and mineral oil $(1: 1, v / v)$ were taken by varying the $T_{R}$ from 300 to 650 $\mathrm{ms}$ at 50 -ms intervals and the $\mathrm{T}_{\mathrm{E}}$ from 13 to $25 \mathrm{~ms}$ at $1-\mathrm{ms}$ intervals with 1-mm-thick slices. Furthermore, the optimum pulse width of the radiofrequency was determined by varying the radiofrequency from $2 \mathrm{kHz}$ to $5 \mathrm{kHz}$. The MR image that discriminated most clearly between water and mineral oil was as follows: $T_{R}=500 \mathrm{~ms}, T_{E}=20 \mathrm{~ms}$, and the pulse width $=4 \mathrm{kHz}$. In addition to these parameters, other parameters were employed for MR imaging of rat brains: the gradient magnetic field, 3 $\mathrm{mT} / \mathrm{cm}$; the fields of view (FOV), $10 \times 10 \mathrm{~cm}^{2}$ for sagittal sections of brains and $6 \times 6 \mathrm{~cm}^{2}$ for coronal sections of brains; the slice thickness, $1.0 \mathrm{~mm}$; and the size of the data matrix, 128 phase-encoded steps. Each image corres- 


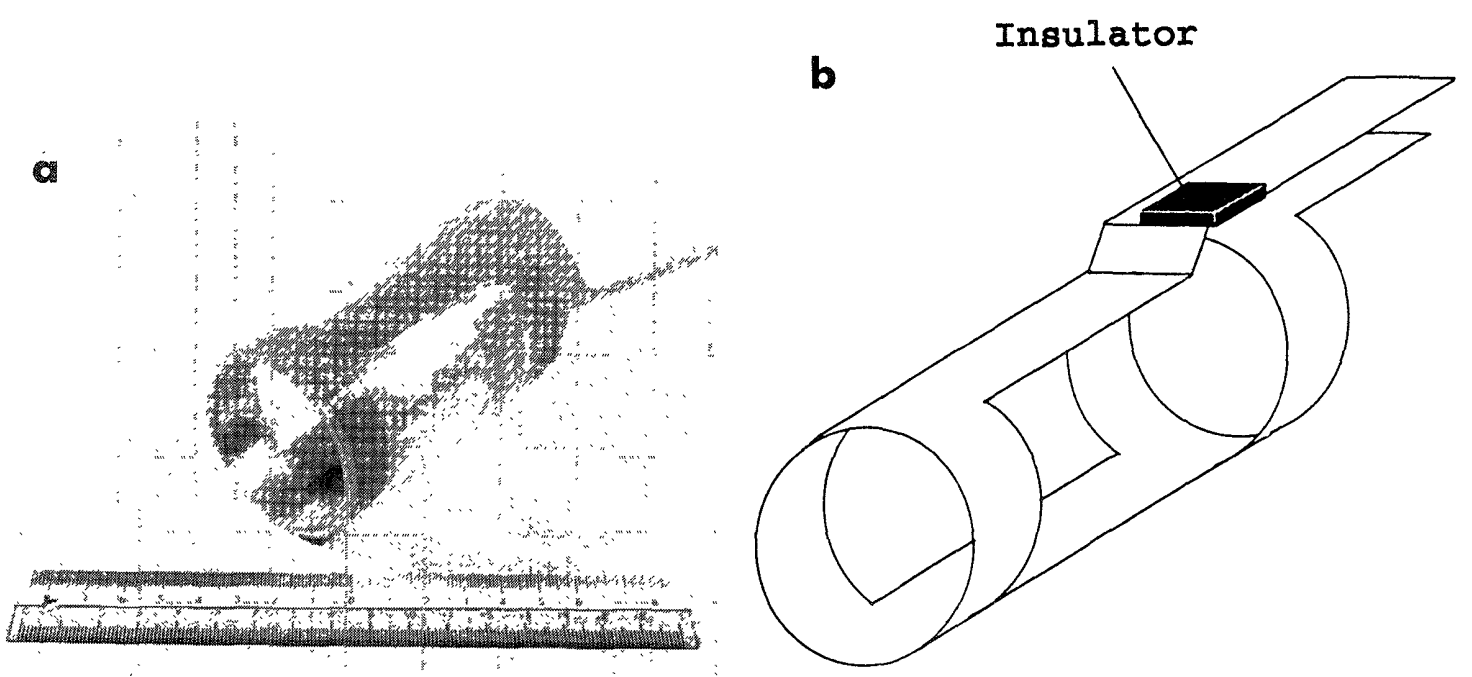

Fig. 1. (a) The imaging probe (Helmholtz coil) was made of thin copper film acording to the Dadok design. An acrylic tube was used as a support for the film. (b) Illustration of the probe. A teflon sheet of adequate thickness was inserted at the junction of two ends of film as an insulator to adjust the probe to resonate at $300 \mathrm{MHz}$.

ponded to 8 acquisitions requiring a total of $512 \mathrm{sec}$.

Rats: Wistar rats (WKAH) were group-housed in a facility accredited by the Guide for the Care and Use of Laboratory Animals of Hokkaido University, School of Medicine with food and water ad libitum. Rats were maintained under a 12:12 h light: dark cycle. Male and female Wistar rats (WKAH) (5 weeks old, $\mathrm{n}=10$ for males and 6 for females; $23-24$ months old, $n=3$ for males and 3 for females) were anesthetized with pentobarbital sodium solution $(40 \mathrm{mg} / \mathrm{kg})$ diluted 2.5 times with a mixed solution of propylene glycol/ethanol/ $\mathrm{H}_{2} \mathrm{O}(2 / 1 / 7$, w/w). The rats were mounted on an acrylic stereotaxic apparatus that extended the length of the probe. Ear bars made of cylindrical acrylic tubes containing mineral oil inside were driven into both auditory canals of the rat. After tuning the probe and adjusting the shim coil, a horizontal section of brain was first imaged. The site of the interaural line, which is defined as the line combining both auditory canals in the brain [10], was recognized as that combining two images of mineral oil on both sides of the head. In this manner the head of the rat could be precisely centered in the magnetic field. The sagittal section of the brain was next imaged to determine the position at $+5.5 \mathrm{~mm}$ from the interaural line. The coronal section was finally imaged at this position.

Semi-microscopic observation of coronal sections: A 5 -week-old male rat was killed by inhaling an overdose of diethylether. The brain was removed and immersed in $10 \%$ neutral buffered formalin. It was then cut into 1-mm-thick coronal slices at about $5 \mathrm{~mm}$ from the interaural line with a microslicer without embedding. Using a stereomicroscope, a photoimage of the slice was taken with transmitted light.

Measurements of signal intensities in MR images: The relative signal intensities in the various regions of MR images were measured on a computer display terminal by using the $0.2 \times 0.2 \mathrm{~mm}^{2}$ box cursor of the computer. The signal intensities were measured at five points in the hypothalamus, cerebral cortex, hippocampus and thalamus in each image, and the means were then calculated. Since little difference in the mean signal intensities between young and aged and/or male and female rats was observed in the hypothalamus, the percent ratios of signal intensities in the cerebral cortex, hippocampus and thalamus to the signal intensity in the hypothalamus were calculated in each image using the following equation.

$$
\text { Ratio }(\%)=\frac{[<\text { SI }>\text { at each region }-<\mathrm{BG}>]}{[<\text { SI }>\text { at hypothalamus }-<\mathrm{BG}>]} \times 100(2)
$$

$<\mathrm{SI}>$ and $<\mathrm{BG}>$ are the means of signal intensities measured at five points in the designated regions and the mean background intensity, respectively.

Measurements of areas on MR images of sagittal sections: Areas of the whole brain, third ventricle and cranial bone were measured by planimeter on a photograph of each MR image. The ratios of the areas of third ventricle and cranical bone to that of the whole brain were calculated.

Statistical analysis: Statistical analyses were made using Student's $t$-test. A probability level of less than $5 \%$ was regarded as significant.

\section{RESULTS}

MR image and semi-microscopic photoimage of coronal sections: The MR image of the coronal section at $+5.5 \mathrm{~mm}$ from the interaural line of the 5-week-old male rat brain was obtained (Fig. 2a) with the probe shown in Fig. 1. After the MR imaging, the brain was removed and immersed in formalin, and a microslice was made at the position around $5 \mathrm{~mm}$ from the interaural line. The slice was photoimaged as shown in Fig. 2b. The illustration of the coronal section at $+5.5 \mathrm{~mm}$ from the interaural line was drawn according to the stereotaxic atlas of Paxions 


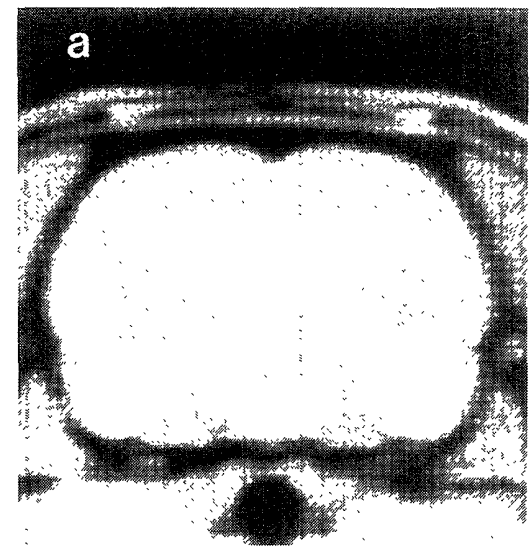

b

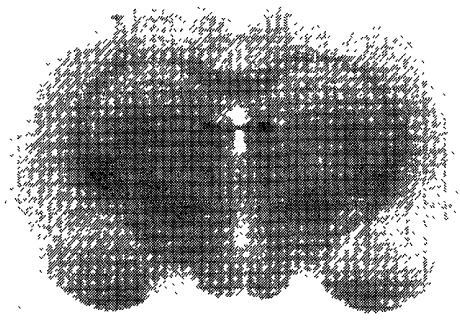

\|\|\|\|\|\|\|\|$\||\||\|\mid\|$

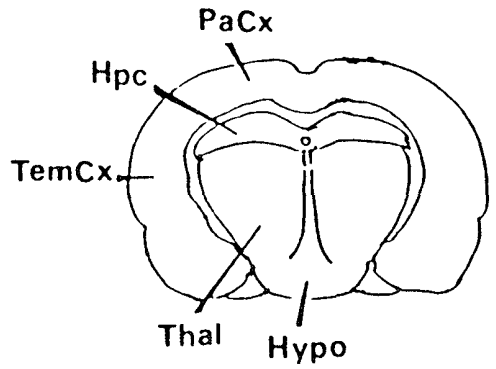

Fig. 2. (a) MR image of the coronal section of a rat brain (male, 5 weeks old) at $+5.5 \mathrm{~mm}$ from the interaural line. (b) Photoimage of a brain slice cut in a $1 \mathrm{~mm}$ thickness at the same position. The interval between two bars corresponds to $1 \mathrm{~mm}$. (c) Illustration of the coronal section at $+5.5 \mathrm{~mm}$ from the interaural line, drawn based on the figure of paxions and Watson

(10). PaCx: parietal cortex, Hpc: hippocampus, TemCx: temporal cortex, Thal: thalamus, Hypo: hypothalamus.
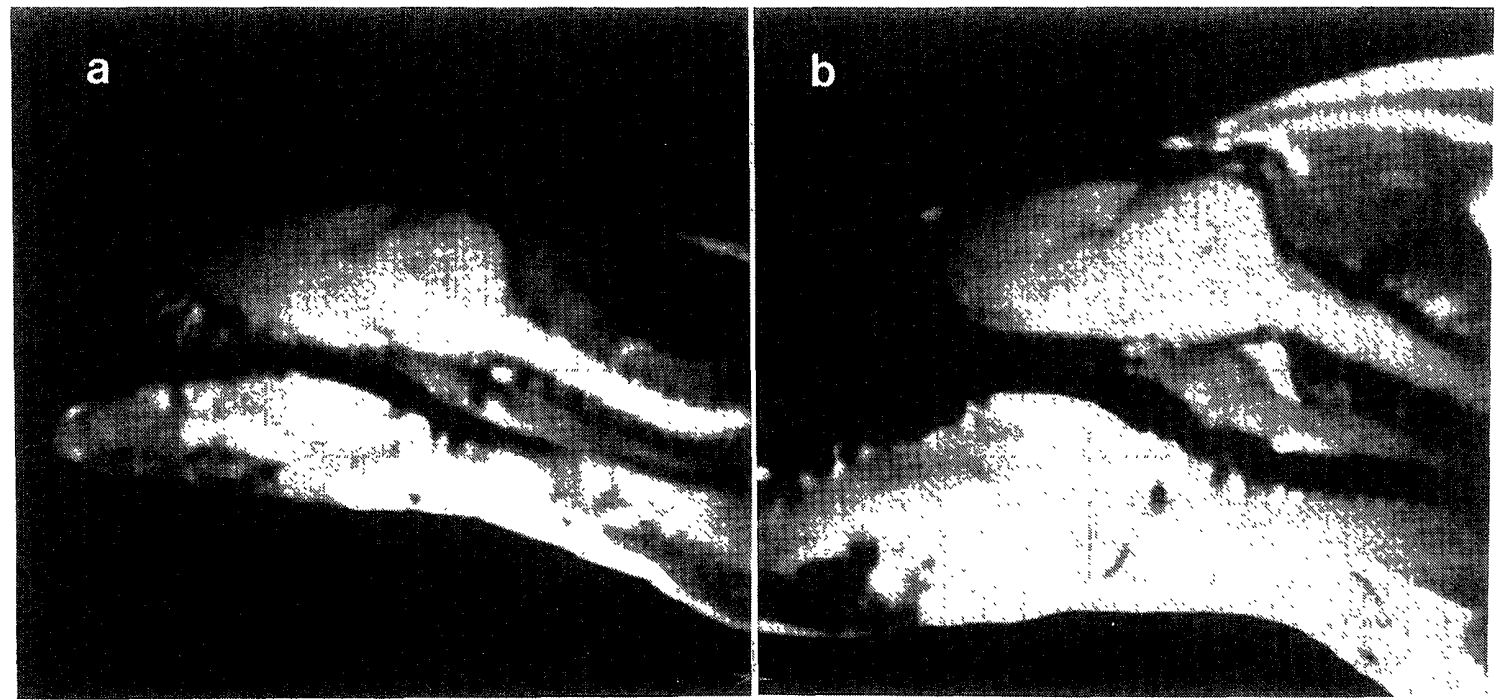

Fig. 3. $\mathrm{T}_{1}$-weighted MR images of sagittal sections of male rat heads (1-mm thick), (a) young (5 weeks old) and (b) aged (23 months old). MR images were taken under a magnetic field of $7.05 \mathrm{~T}$ by employing the following conditions. $\mathrm{T}_{\mathrm{R}}=$ $500 \mathrm{~ms}, \mathrm{~T}_{\mathrm{E}}=20 \mathrm{~ms}, \mathrm{FOV}=6 \times 6 \mathrm{~cm}^{2}, 128$ phase encodes, 8 acquisitions, pixel size $=0.2 \times 0.2 \mathrm{~mm}^{2}$.

and Watson [10] (Fig. 2c). Each region is indicated in this illustration. Comparison of the MR image and photoimage revealed that the cerebral cortex, hippocampus, hypothalamus and thalamus were clearly imaged by this probe.

MR images of sagittal sections of young and aged rat brains: MR images of the sagittal sections at the midlines of the brains of young ( 5 weeks old) and aged ( 23 months old) male rats are shown in Figs. $3 a$ and $3 b$, respectively. The two figures show that the whole-brain size changed little in both groups. The image of the whole brain was uniform in the young rat and some scatter of NMR intensities in MR images was present in the aged rat. The third ventricle was clearly imaged, and its enlargement was observed in aged rats. Furthermore, marked hypertrophy of the parietal bone was observed in aged rats. The average thickness of parietal bones in ten young rats was
$0.3 \mathrm{~mm}$, whereas that of three aged rats was $1.5 \mathrm{~mm}$.

To quantitatively confirm these observations, the area of the third ventricle $v s$ the area of the whole brain and the area of parietal bone $v s$ the area of the whole brain were calculated. The results were expressed as percent ratios and are shown in Fig. 4a. Differences in the areas of the third ventricles and parietal bones between young and aged rats were statistically significant at $* 0.01<\mathrm{p}<0.05$ and $* * \mathrm{p}<0.01$, respectively.

MR images of sagittal sections of young (5 weeks old) and aged ( 24 months old) female rat brains were quite similar to those of male rats (data not shown). The hypertrophy of parietal bones was observed in aged female rats $(0.3-\mathrm{mm}$ thick in 3 rats to $1.2 \mathrm{~mm}$ in 6 rats). Differences in the areas of the third ventricles and parietal bones between young and aged female rats were statistically significant at $* 0.01<\mathrm{p}<0.05$ and $* * \mathrm{p}<0.01$, respec- 


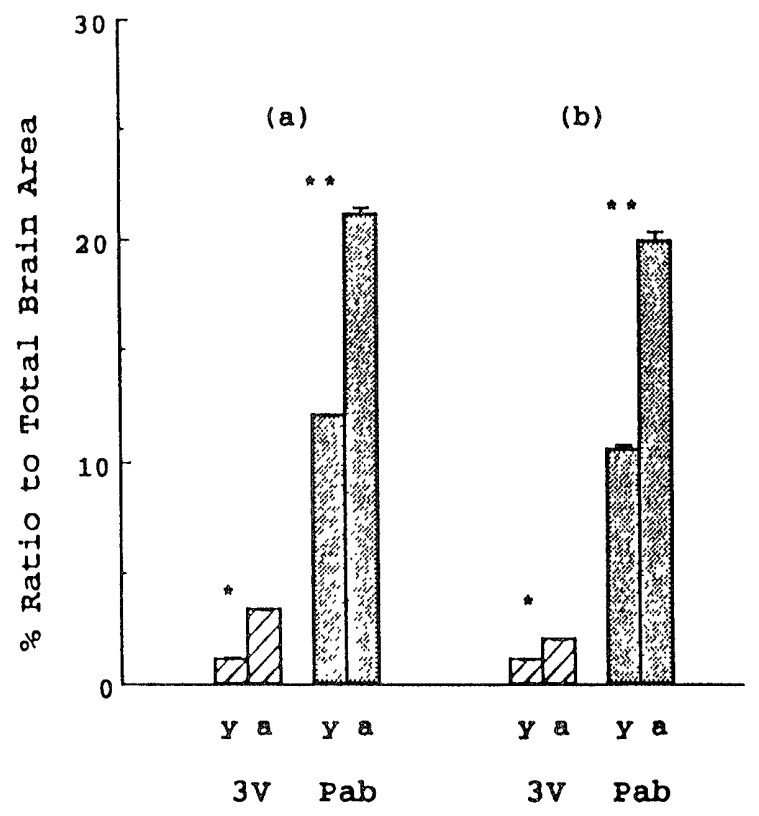

Fig. 4. (a) Percent ratios of areas of the third ventricles (left) and parietal bones (right) to those of whole brains in young and aged male rats. (b) Percent ratios of areas of th third ventricles (left) and parietal bones (right) to those of whole brains in young and aged female rats. The differences in these ratios between the rats were statistically significant $\left({ }^{*} 0.01<\mathrm{p}<0.05,{ }^{* *} \mathrm{p}<0.01\right)$.

tively (Fig. 4b).

When the areas of both third ventricles and parietal bones in male rats were compared to those in female rats, the enlargement of third ventricles and hypertrophy of parietal bones in aged male rats was found to be more marked than in aged female rats $\left({ }^{*} p<0.05\right)$. No statistically significant differences in the areas between males and females were observed in young rats.
$M R$ images of coronal sections of young and aged rat brains: MR images of coronal sections at $+5.5 \mathrm{~mm}$ from the interaural line in brains of young and aged male rats are shown in Figs. 5a and 5b, respectively. The cerebral cortex, hippocampus, hypothalamus and thalamus could be imaged in young male rats. The most intense NMR signals were observed at the hypothalamus region. The average thickness of parietal bones was $0.3 \mathrm{~mm}$ (10 rats). Aged male rats also gave the most intense NMR signals in the hypothalamus region, a result similar to that obtained in young rats. However, the NMR intensities in the cerebral cortex, hippocampus and thalamus tended to decrease in aged rats. The average thickness, $1.5 \mathrm{~mm}$, of parietal bones ( 3 rats) was the same as that observed in the sagittal sections of aged rats.

These observations were quantitatively confirmed by calculating the ratios of the mean signal intensities from the cerebral cortex, hippocampus and thalamus against that from the hypothalamus in each image according to equation (2). The results obtained from male rats were expressed as percent ratios and are shown in Fig. 6a. Differences in the signal intensities between cerebral cortices of young and aged rats and between thalami of young and aged animals were significantly different at $* \mathrm{p}<0.05$.

MR images of the coronal sections at $+5.5 \mathrm{~mm}$ from the interaural line in brains of young ( 5 weeks old) and aged (24 months old) female rats were similar to those obtained from male rats (data not shown). However, the decreases in the NMR intensities of the cerebral cortex, hippocampus and thalamus with aging in females were not as marked as those in males. Hypertrophy of the parietal bone was recognized $(0.3 \mathrm{~mm}$ in 6 rats to $1.2 \mathrm{~mm}$ in 3 rats), but its extent was also less than that in males. The quantitative evaluation of MRI images according to equation (2) showed that a statistically significant difference $\left({ }^{*} p<0.05\right)$ was present only between cerebral cor-
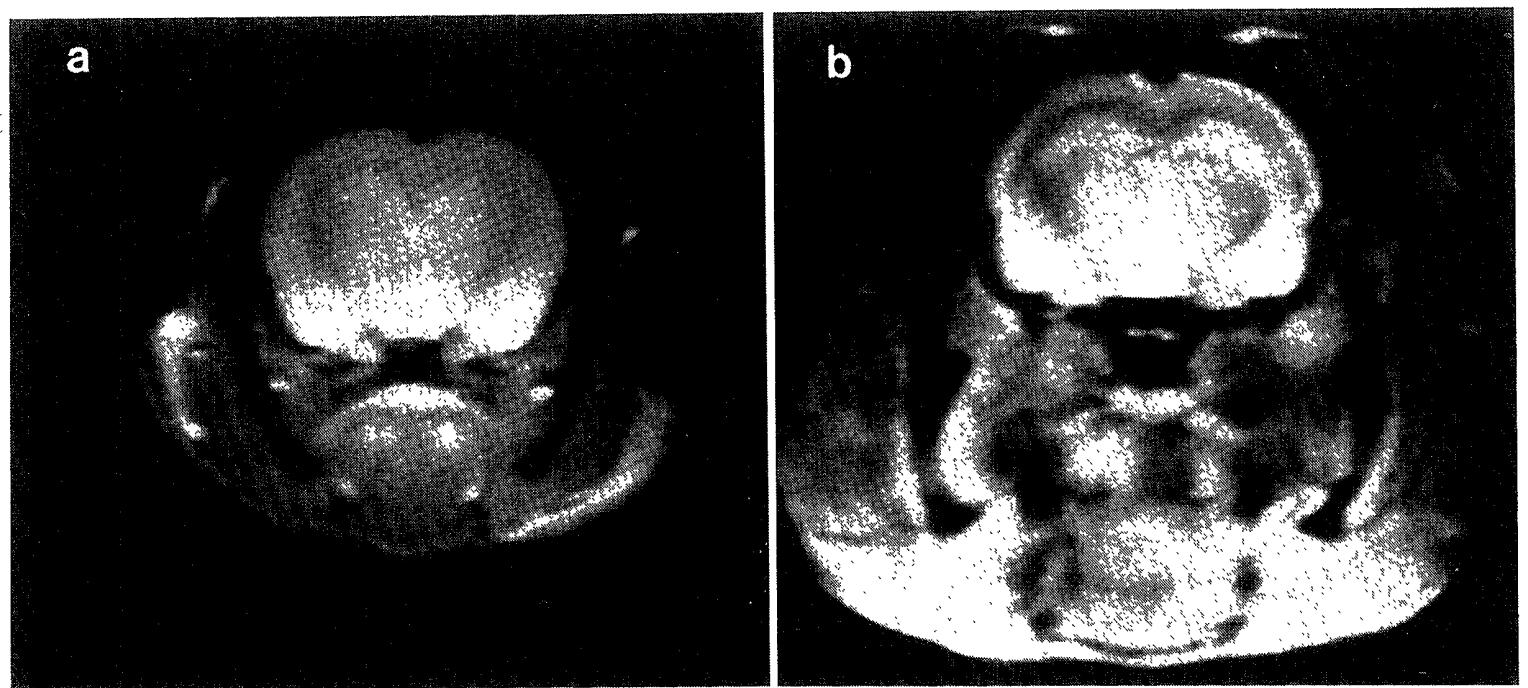

Fig. 5. $T_{1}$-weighted MR images of coronal sections of male rat heads (1-mm thick) at $+5.5 \mathrm{~mm}$ from the interaural line, (a) young ( 5 weeks old) and (b) aged ( 23 months old). MR images were taken under the similar conditions to Fig. 3 except for the pixel size $\left(0.12 \times 0.12 \mathrm{~mm}^{2}\right)$. 
(a)

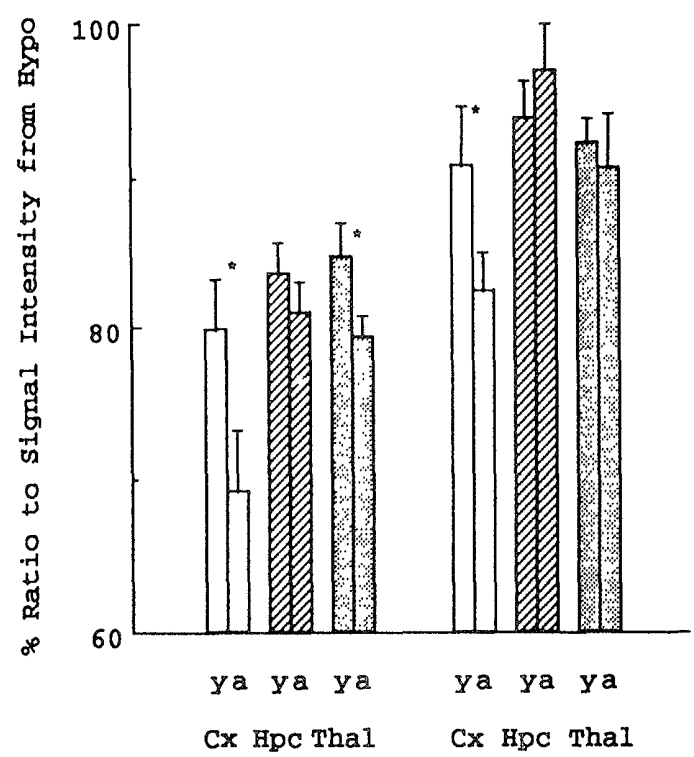

Fig. 6. Percent ratios of MR signal intensities of the cerebral cortex, hippocampus and thalamus to that of the hypothalamus in young and aged male (a) and female (b) rats. Statistically significant differences between young and aged rats were observed in the cerebral cortex and thalamus in male rats and only in the cercbral cortex in female rats $\left({ }^{*} 0.01<\mathrm{p}<0.05\right)$.

tices of young and aged rats (Fig. 6b).

DISCUSSION

In the present study differences between the MR images of young and aged rat brains were observed. Irrespective of the difference between the sexes, hypertrophy of parietal bones and enlargement of the third ventricles were clearly observed in aged rats. The enlargement was regarded as a reflection of brain atrophy accompanying aging $[12,13,15]$. From the fact that the enlargement of the third ventricle in aged rats was slightly more marked in males than in females, it was inferred that greater histological alterations of brains occurred in males than in females. Condon et al. reported that there was a significant decrease in brain volume between the ages of 20 and 60 years in human males, whereas the decrease was not statistically significant in human females [2].

The decrease of NMR signal intensities in the cerebral cortices in aged (especially male) rats might be explained by two reasons: 1 ) the change of lipid components to substances giving low signal intensities due to lipid peroxidation [11], 2) a decrease in the absolute number of nervous cells together with a decrease in the number of hydrogen nuclei, resulting in the low intensities of NMR signals (see equation (1)). Decreases in the number of nervous cells were actually found in brains of monkeys, dogs, cats, and rats [15]. It should be noted at this point that few histological alterations with aging were observed in the hypothalami of either male or female rats.
In the present study a Helmholtz-type MRI probe was made to obtain MR images of brains of young and aged rats under a magnetic field of $7.05 \mathrm{~T}$. Histological alterations in cerebral cortices, third ventricles and parietal bones in aged rats were clearly imaged by this probe. These observations lead us to conclude that this probe is applicable for the logitudinal study of small laboratory animals.

ACKNOWLedgements. We thank Rooibos Tea Japan Co., Ltd., for financially supporting us. This work was also supported in part by a Grant-in-Aid for Scientific Research Fund from the Ministry of Education, Science and Culture of Japan (\#05556051).

\section{REFERENCES}

1. Araki, T. 1984. pp. 27-31. NMR-CT. Nankodo, Tokyo (in Japanese).

2. Condon, B., Grant, R., Hadley, D., and Lawrence, A. 1988. Brain and intracranial cavity volumes: in vivo determination by MRI. Acta Neurol. Scand. 78: 387-393.

3. Crooks, L. E., Mills, C., and Davis, P. L. 1982. Visualization of cerebral and vascular abnormalities by NMR imaging. The effects of imaging parameters on contrast. Radiology 144: 843-852.

4. de la Torre, J. C., Fortin, T., Park, G. A. S., Butler, K. S., Kozlowski, P., Pappas, B. A., de Socarraz, H., Saunders ,J. K., and Richard, M. T. 1992. Chronic cerebrovascular insufficiency induced dementia-like deficits in aged rats. Brain Res. 582: 186-195.

5. Dixon, R. L. and Ekstrand, K. E. 1982. The physics of proton NMR. Med. Phys. 9: 807-818.

6. Elster, A. D. 1988. An index for comparative parameter weighing in MR imaging. J. Comput. Assist. Tomogr. 12: 130-134.

7. Gardian. D. G. 1982. Nuclear Magnetic Resonance and Its Applications to Living Systems, Chapter 8. Oxford University Press, New York.

8. Ishige, N., Pitts, L. H., Berry, I., Carlson, S. G., Nishimura, M. C., Moseley, M. E., and Weinstein, P. R. 1987. The effect of hypoxia on traumatic head injury in rats: Alterations in neurologic function, brain edema, and cerebral blood flow. J. Cereb. Blood Flow Metabol. 7: 759-767.

9. London, R. E., Tony, G., Gabel, S. A., and Funk, A. 1989. Magnetic resonance imaging studies of the brains of anethetized rats treated with manganese chloride. Brain Res. Bull. 23: 229-235.

10. Paxions, G. and Watson, C. 1982. The Rat Brain in Stereotaxic Coordinates, Figure 22. Academic Press, Sydney.

11. Pettegrew, J. W., Panchalingam, K., Withers, D. G., McKeag, D., and Strychor, M. P. H. 1990. Changes in brain energy and phospholipid metabolism during development and aging in the Fischer 344 rat. J. Neuropathol. Exp. Neurol. 49: 237-249.

12. Pfefferbaum, A., Zatz, L. M., and Jernigan, T. L. 1986. Computer-interactive method for quantifying cerebrospinal fluid and tissue in brain CT scans: Effects of aging. $J$. Comput. Assist. Tomogr. 10: 571-578.

13. Shimada, A., Ohta, A., Akiguchi, I., and Takeda, T. 1992. Inbred SAM-P/10 as a mouse model of spontaneous, 
inherited brain atrophy. J. Neuropathol. Exp. Neurol. 51: 440-450.

14. Takahashi, M., Fritz-Zieroth, B., Yamaguchi, M., Ogawa, H., Tanaka, T., Sasagawa, S., Chiguro, T., Ohta, Y., and Okamoto, K. 1992. MR imaging of cerebral lesions accompanying stroke in stroke-prone spontaneously hypersensi- tive rats. Folia Pharmacol. Japon 100: 21-28 (in Japanese with English abstract).

15. Tomonaga, M. 1989. Shinkeikei no Kareihenka. pp. 1-46. In: Nou Shinkeikei no Eiging (Tomonaga, M. and Sato, A. eds.), Asakurashoten, Tokyo (in Japanese). 